\title{
Cambios en el clima local y su efecto en la regulación hídrica en microcuencas del departamento del Magdalena, Norte de Colombia
}

\author{
José E. Revueltas ${ }^{1}$, Alvaro Zabaleta ${ }^{1}$, Teobaldis Mercado ${ }^{2 *}$ y Sonia Aguirre ${ }^{3}$ \\ (1) Facultad de ingeniería, Universidad de Córdoba, Carrera 6 No 77 - 305. CP. 230002. Montería, Córdoba, Colombia \\ (correo-e: joserevueltas94@gmail.com; alvarozabaleta92@gmail.com). \\ (2) Facultad de Ciencias Agrícolas, Universidad de Córdoba, Carrera 6 No 77 - 305. CP. 230002. Montería, Córdoba, \\ Colombia (correo-e: tmercado@correo.unicordoba.edu.co). \\ (3) Universidad de Magdalena, Carrera 32 No 22 - 08. CP- 470004. Santa Marta, Magdalena, Colombia \\ (correo-e: saguirre@unimagdalena.edu.co).
}

${ }^{*}$ Autor a quien debe ser dirigida la correspondencia

Recibido Jul. 13, 2020; Aceptado Sep. 11, 2020; Versión final Oct. 15, 2020, Publicado Dic. 2020

\section{Resumen}

El objetivo de esta investigacion es identificar posibles manifestaciones de cambios en el clima local en 13 microcuencas en el Norte de Colombia mediante el análisis estadístico de la temperatura, humedad atmosférica, densidad de vapor y evapotranspiración potencial. Se estimó la respuesta hidrológica del territorio para inferir la afectación en la oferta hídrica utilizando el método del número de curva. Los resultados muestran un incremento promedio de $0.66^{\circ} \mathrm{C}$ en la temperatura del aire. La densidad de vapor de agua atmosférico alcanzó un aumento cercano a 16\% y la evapotranspiración potencial presenta un incremento promedio de $30.9 \mathrm{~mm}$. En 11 microcuencas se encontraron números de curva mayores de 80 en más del $85 \%$ de su territorio. Se concluye que las tendencias de posible cambio en el clima local están relacionadas con el estado físico del territorio en términos de cobertura vegetal, como principal regulador hídrico y térmico.

\section{Changes in local climate and its effects on micro-basins water regulation in Magdalena, Northern Colombia}

\begin{abstract}
The primary objective of this research study is to identify possible manifestations of local climate change in 13 micro-basins in Northern Colombia through statistical analyses of temperature, atmospheric humidity, vapor density, and potential evapotranspiration. The hydrological response area is estimated to infer the effect on water supply using the curve number method. The results show an average increase of $0.66^{\circ} \mathrm{C}$ in air temperature. Atmospheric water vapor density reached an increase close to $16 \%$, whereas potential evapotranspiration shows an average increase of $30.9 \mathrm{~mm}$. Curve numbers greater than 80 are found in eleven micro-basins in more than $85 \%$ of their territories. It is concluded that trends of potential local climate change are related to the area's physical state in terms of vegetation cover, as the main water and thermal regulator.
\end{abstract}

Keywords: climate variables; curve number; surface physical state; runoff; hydrological response 


\section{INTRODUCCIÓN}

El calentamiento del sistema climático global es una realidad y se evidencia en el incremento de la temperatura promedio del aire y océanos, el deshielo y el aumento del nivel del mar. Los procesos de calentamiento y enfriamiento de la superficie terrestre ocurren de manera natural, debido a numerosas causas. Estos procesos han presentado alteraciones observables desde hace varias décadas, representando un aumento anómalo en la temperatura global como resultado de la acumulación en la atmósfera de gases de efecto invernadero, los cuales absorben la mayor parte de la energía infrarroja emitida por la tierra, dando como resultado el calentamiento de la superficie y cambios en el clima global (IPCC 2007). El fenómeno de calentamiento global, implica modificaciones del ciclo hidrológico y evidencia de variaciones importantes en el régimen de precipitación que afectan negativamente la oferta hídrica directa para zonas del occidente de Sur América, pudiéndose presentar sequías y estrés hídrico (Bryson et al., 2008). En efecto, el cambio climático, expresado en la modificación de la temperatura media, la precipitación anual, la humedad relativa y la escorrentía, conllevara a transformaciones para el ciclo hidrológico y, por ende, se tendrán efectos importantes sobre el recurso hídrico y la disponibilidad de agua (Alarcón et al., 2019)

A nivel mundial para atender desde un punto de vista científico el cambio climático se ha estudiado los aumentos de la temperatura del aire, variaciones en el contenido de humedad del aire y cambios en los regímenes de lluvias. Roberto y Oregon (2004) utilizan la temperatura y la evapotranspiración de Thornthwaite (modificada) para la descripción y análisis de climas áridos. Gónima (2009) muestra evidencias del calentamiento global en el Caribe colombiano, representadas por variaciones de la temperatura del aire, la precipitación, la evapotranspiración y el déficit hídrico. Kousari et al. (2011) analizaron las tendencias de cambio mensuales y anuales en las temperaturas mínimas, máximas y medias, humedad relativa y la precipitación en 26 estaciones climáticas en Irán para un periodo de 55 años de registro. Zhang et al. (2020) seleccionaron las tendencias temporales de evapotranspiración potencial y precipitación como indicadores de cambio climático, establecieron cinco escenarios posibles y se examinaron las relaciones entre la desertificación y los indicadores de cambio climático para diferentes períodos de tiempo en el norte de China.

La temperatura, el contenido de vapor de agua atmosférico y la evapotranspiración potencial de la interface suelo - vegetación, son una medida de cambios en el clima local en el trópico, cuyas características corresponden a una alta temperatura y humedad relativa, resultando como indicadores de la manifestación de calentamiento global a escala local de la superficie (Camuffo 2002; Gónima y Pérez 2012; New, Hulme, y Jones 1999, 2000; Pérez 2015; Pérez y Gónima 2014). En el estudio hidroclimático la cobertura vegetal es fundamental en los procesos de retención hídrica del suelo, estabilización de la temperatura y control en la dinámica de escurrimiento superficial por su relación en los procesos de abstracción. Lo anterior implica que la condición de cobertura del suelo, se convierta en un indicador hidrológico de la posible manifestación de calentamiento global a escala local, permitiendo relacionarla con la respuesta hidrológica de una zona mediante la estimación de escurrimientos y balances hídricos que involucran las variables termodinámicas atmosféricas (FAO 2006).

En los últimos años se han desarrollado estudios que relacionan el estado físico de la cobertura del terreno y la respuesta hidrológica del mismo bajo los escenarios de calentamiento global. Zabaleta (2017) realizó un estudio hidrológico con enfoque climático que involucra el análisis de variables climáticas y su relación en la disponibilidad hídrica en la Zona de Sabana departamento de Córdoba, Colombia; así mismo Zabaleta et al. (2018) analizaron el comportamiento hidrológico de la superficie de la cuenca baja de los afluentes del Cauca, San Jorge y del río Magdalena en la llamada región de La Mojana, en el Norte de Colombia. Napoli et al. (2017) analizaron la respuesta hidrológica en la cuenca del rio Elsa ante los cambios en el uso del suelo y cambios climáticos durante el periodo comprendido entre 1954 y 2007, mediante el modelo ArcSWAT. Correia et al. (2007) utilizaron el modelo regional numérico (Eta) junto con el Modelo Simplificado de Biosfera Simple (SSiB) para investigar el impacto de los cambios en la cubierta terrestre en el clima regional en la Amazonía. Shiferaw et al. (2018) evaluaron la generación de escorrentía superficial bajo escenarios de cambio climático para la cuenca de llala en las tierras altas del norte de Etiopía. La presente investigación identificó las posibles manifestaciones de cambio del clima local mediante el estudio de variables climáticas, y analizó la respuesta hidrológica del territorio para inferir la afectación en la oferta hídrica.

\section{MATERIALES Y MÉTODOS}

La zona de estudio comprende un área de $10615.22 \mathrm{Km}^{2}$ de 13 microcuencas distribuidas entre los municipios Zona Bananera, Chivolo, Sabanas de San Ángel, Santa Barba de Pinto, Plato, Nueva Granada, Guamal y Sitio Nuevo, en el departamento de Magdalena, Colombia (figura 1). En el municipio de Sitio Nuevo se tuvo en cuenta el área tributante a la Ciénaga grande del Magdalena. De forma general, en la zona de estudio la precipitación presenta un valor promedio de $1317.1 \mathrm{~mm}$ anuales con variaciones entre $791.1 \mathrm{~mm}$ y los 1843.1 $\mathrm{mm}$, la temperatura presenta valor promedio de $28.2^{\circ} \mathrm{C}$ con un intervalo de confianza entre los $27.5^{\circ} \mathrm{C}$ y los 
$29.0^{\circ} \mathrm{C}$, en tanto que la humedad relativa está en un intervalo de confianza entre el $77 \%$ y el $82 \%$, con valor promedio de $79 \%$.

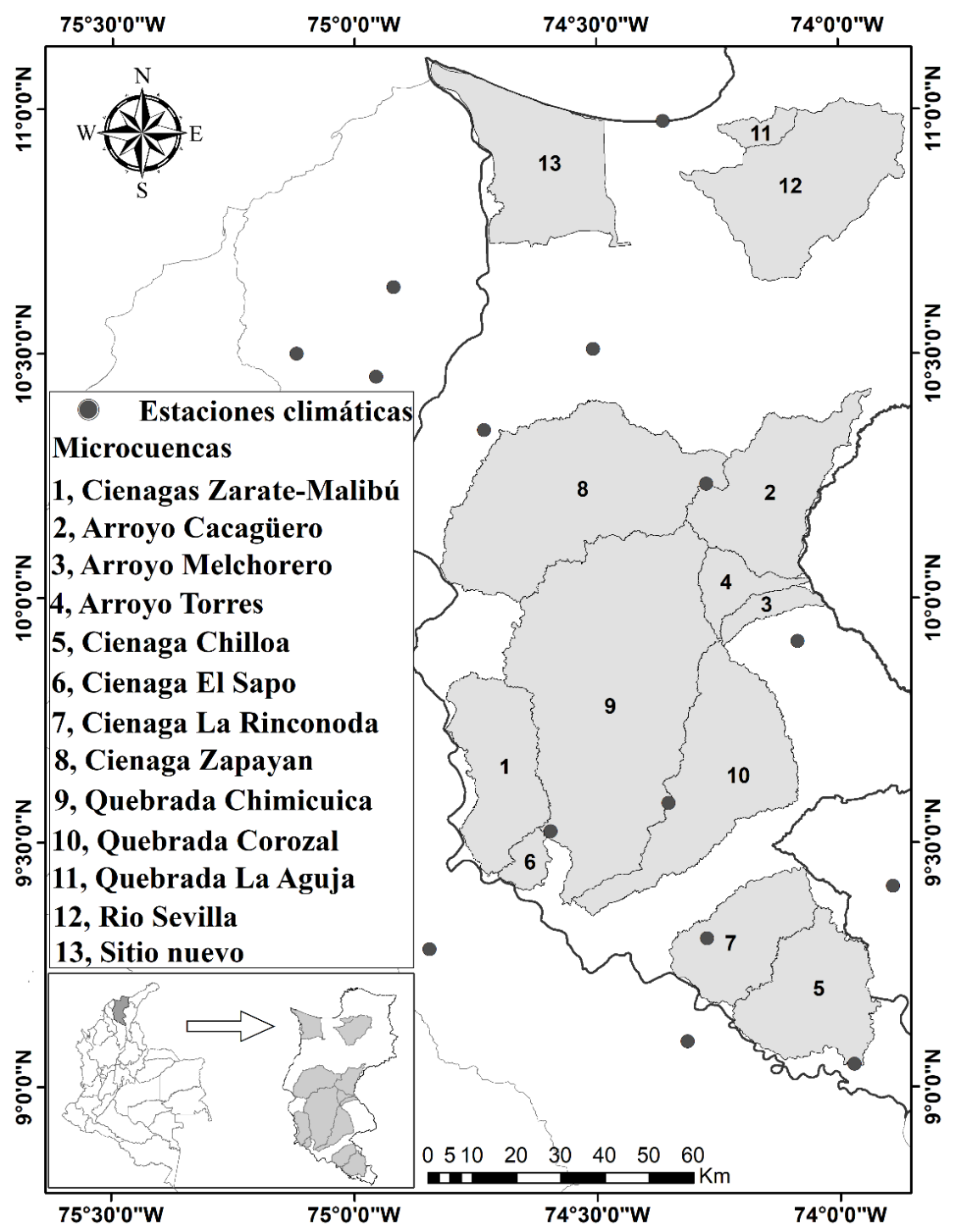

Fig. 1: Localización microcuencas estudiadas en el departamento del Magdalena

\section{Identificación de cambio en el clima}

La identificación del cambio en el clima local, se realizó con base en el modelo metodológico elaborado por Pérez (2015), el cual consistió en el análisis estadístico de la temperatura y humedad atmosférica, cálculo de densidad de vapor y evapotranspiración potencial de la interface suelo vegetación, análisis de la variación multianual, a partir de ecuaciones de regresión para hallar la tendencia de cambio en el periodo 1985 - 2016. La información climática del área de estudio fue suministrada por el Instituto de Hidrología, Meteorología y Estudios Ambientales (IDEAM), para 16 estaciones climáticas; siendo 5 climatológicas principales y 11 pluviométricas (tabla 1).

Inicialmente se evaluó la calidad de la serie de datos climáticos suministrados mediante análisis de promedios, diferencias y desviaciones estándar, para detectar datos extremos atípicos o anómalos que pudieran alterar significativamente el comportamiento de las series por errores de registro o calibración de equipos de medición empleados para tal fin, seguidamente se realizaron análisis de rachas, test de persistencia $\mathrm{R}$ y test de Helmert para determinar la aleatoriedad, homogeneidad y persistencia. Una vez realizada la verificación estadística de la información suministrada por el IDEAM, los datos faltantes fueron calculados mediante regresión simple lineal para temperatura T y medias móviles y regresión lineal para humedad relativa HR. La generación de datos faltantes de HR en estaciones con baja calidad en la información al no conseguir resultados significativos en la verificación estadística, se realizó regresión por regionalización toda vez que se verificó la correspondencia de régimen climático en las estaciones. Posteriormente se evaluó la calidad de las 
series con información calculada mediante los procedimientos anteriormente descritos incluyendo en dicha verificación los métodos de correlación de Spearman y simple lineal.

Tabla 1: Estaciones climáticas del área de estudio.

\begin{tabular}{|c|c|c|c|c|c|}
\hline Estación & Latitud & Longitud & Estación & Latitud & Longitud \\
\hline Apto Baracoa & $9^{\circ} 16^{\prime} 55^{\prime \prime} \mathrm{N}$ & $74^{\circ} 50^{\prime} 43^{\prime \prime} \mathrm{O}$ & El Canal & $9^{\circ} 24^{\prime} 37^{\prime \prime} \mathrm{N}$ & $73^{\circ} 53^{\prime} 25^{\prime \prime} \mathrm{O}$ \\
\hline Repelon & $10^{\circ} 30^{\prime} 1 " \mathrm{~N}$ & $75^{\circ} 7^{\prime} 9 " \mathrm{O}$ & El Pueblito & $9^{\circ} 34^{\prime} 52^{\prime \prime} \mathrm{N}$ & $74^{\circ} 21^{\prime} 8^{\prime \prime} \mathrm{O}$ \\
\hline Normal Manati & $10^{\circ} 27^{\prime} 12^{\prime \prime} \mathrm{N}$ & $74^{\circ} 57^{\prime} 16^{\prime \prime} \mathrm{O}$ & Tio Gollo & $10^{\circ} 20^{\prime} 39^{\prime \prime} \mathrm{N}$ & $74^{\circ} 43^{\prime} 55^{\prime \prime} \mathrm{O}$ \\
\hline Los Alamos & $9^{\circ} 18^{\prime} 14^{\prime \prime} \mathrm{N}$ & $74^{\circ} 16^{\prime} 25^{\prime \prime} \mathrm{O}$ & El Guamo & $10^{\circ} 2^{\prime} 14^{\prime \prime} \mathrm{N}$ & $74^{\circ} 58^{\prime} 23^{\prime \prime} \mathrm{O}$ \\
\hline Santa Rosa & $9^{\circ} 5^{\prime} 36^{\prime \prime} \mathrm{N}$ & $74^{\circ} 18^{\prime} 50^{\prime \prime} \mathrm{O}$ & Sabanalarga & $10^{\circ} 38^{\prime} 12^{\prime \prime} \mathrm{N}$ & $74^{\circ} 55^{\prime} 8^{\prime \prime} \mathrm{O}$ \\
\hline La Mecha & $9^{\circ} 31^{\prime} 22 " \mathrm{~N}$ & $74^{\circ} 35^{\prime} 44^{\prime \prime} \mathrm{O}$ & Apto Las Flores & $9^{\circ} 2^{\prime} 46^{\prime \prime} \mathrm{N}$ & $73^{\circ} 58^{\prime} 15^{\prime \prime} \mathrm{O}$ \\
\hline Monterrubio & $10^{\circ} 14^{\prime} 1 " \mathrm{~N}$ & $74^{\circ} 16^{\prime} 23^{\prime \prime} \mathrm{O}$ & El Camp Dificil & $9^{\circ} 54^{\prime} 43^{\prime \prime} \mathrm{N}$ & $74^{\circ} 5^{\prime} 8^{\prime \prime} \mathrm{O}$ \\
\hline Tasajera & $10^{\circ} 58^{\prime} 34^{\prime \prime} \mathrm{N}$ & $74^{\circ} 21^{\prime} 42^{\prime \prime} \mathrm{O}$ & Media Luna & $10^{\circ} 30^{\prime} 36^{\prime \prime} \mathrm{N}$ & $74^{\circ} 30^{\prime} 24^{\prime \prime} \mathrm{O}$ \\
\hline
\end{tabular}

Una vez verificada la calidad de las nuevas series de datos, se calcularon los promedios mensuales y anuales multianuales para T y HR. Con los promedios anuales multianuales se calculó la evapotranspiración potencial ETP y la densidad de vapor $d_{v}$ y se evaluó la variabilidad climática mediante regionalización de las 5 estaciones meteorológicas principales, análisis de influencia del fenómeno de oscilación del Sur (ENSO, por sus siglas en inglés) a través de la comparación de la precipitación y la temperatura promedio de los años ENSO con el rango de desviación de las series históricas. El análisis de tendencia para $T, d_{v}$ y HR, este último se realizó cualitativamente mediante regresión simple. El cálculo de $\mathrm{d}_{\mathrm{v}}$ se realizó mediante la expresión:

$$
d_{v}=\frac{t_{v}}{R_{v} T k}
$$

Donde Tk es la temperatura en grados Kelvin $(K), R_{v}$ es la constante específica de vapor de agua (461.8 $\frac{\mathrm{J}}{\mathrm{kg}^{*} \mathrm{~K}}$ ), $t_{v}$ es la tensión de vapor de agua en la atmósfera calculada de la siguiente manera:

$$
t_{v}=\frac{H R}{100} T_{s}
$$

De donde HR es la humedad relativa y $T_{s}$ es la tensión de vapor de agua para una condición de saturación estimada de acuerdo con la siguiente expresión (Leckner, 1978):

$$
\mathrm{T}_{\mathrm{s}}=\exp \left(26.23-\frac{5416}{\mathrm{~T}}\right)
$$

La evapotranspiración potencial (ETP) sin corregir se estimó de acuerdo con Thorthwaite mediante la expresión:

$$
\text { etp }=16\left(\frac{10 t}{\mathrm{l}}\right)^{\mathrm{a}}
$$

Donde etp es la evapotranspiración sin corregir, $t$ es la temperatura promedio mensual, a es un factor de potencia $\left(a=0.492+(0.179 * \mid)-\left(0.0000771^{*} I^{2}\right)+\left(0.000000675^{*} l^{3}\right)\right)$ e I el índice de calor anual calculado mediante la expresión:

$$
\mathrm{I}=\sum_{\mathrm{i}=1}^{12} \mathrm{i}_{\mathrm{i}}
$$

Donde i es el índice de calor mensual, calculado mediante la expresión:

$$
\mathrm{i}=\left(\frac{\mathrm{t}_{\mathrm{i}}}{5}\right)^{1.514}
$$


La ecuación 4, representa la evapotranspiración potencial sin corregir, de manera que esta es ajustada de acuerdo la latitud y longitud mediante un factor de corrección K estimado mediante la ecuación 7, donde "N" es el número máximo de horas de brillo solar en cada localidad y "d" el número de días del mes.

$$
\mathrm{K}=\frac{\mathrm{N}^{*} \mathrm{~d}}{360}
$$

Finalmente, la ETP corregida se calcula teniendo en cuenta dicho factor de corrección $\mathrm{K}$ como:

$$
\mathrm{ETP}=\mathrm{K}^{*} \text { etp }
$$

\section{Determinación de la cobertura vegetal}

Se determinó la cobertura actual del terreno por clasificación digital de imágenes satelitales mediante clasificación supervisada para una imagen Landsat 8 corregida atmosféricamente, tomada el 22 de enero de 2019 y obtenida a través del portal https://earthexplorer.usgs.gov/ del Servicio Geológico de Estados Unidos (USGS, por sus siglas en inglés) que contiene el área de estudio, empleando la metodología Corine Land Cover adaptado para Colombia por el IDEAM. Se determinó el factor de índice óptimo (OIF) para la escogencia de la combinación de tres bandas con mejor separabilidad estadística sobre un total 120 posibles combinaciones, usando 6 bandas (bandas $2,3,4,5,6$, y 7) sobre las cuales se realizó la agrupación. El OIF se determinó mediante la expresión:

$$
\mathrm{OIF}=\frac{\sum \mathrm{S}^{2}}{\sum \mathrm{r}}
$$

Donde $S^{2}$ la desviación estándar de cada banda individual y $r$ es el coeficiente de correlación entre las tres bandas a combinar obtenida a partir de la matriz de correlación.

La evaluación estadística se realizó mediante matriz de confusión usando la comparación entre las condiciones reales del terreno y la clasificación realizada, para ello se hizo un muestreo aleatorio estratificado garantizando que la clasificación realizada representa adecuadamente los distintos tipos de cobertura, se calcularon los parámetros de exactitud del producto, la exactitud del usuario, la exactitud global de la clasificación definido como la relación entre la suma de la diagonal principal de la matriz con el total de puntos muestreados y finalmente se determinó el coeficiente kappa mediante la expresión:

$$
\mathrm{k}=\frac{\mathrm{N} \sum_{\mathrm{i}=1}^{\mathrm{r}} \mathrm{X}_{\mathrm{ii}}-\sum_{\mathrm{i}=1}^{\mathrm{r}} \mathrm{X}_{\mathrm{i}+} \mathrm{X}_{+\mathrm{i}}}{\mathrm{N}^{2}-\sum_{\mathrm{i}=1}^{\mathrm{r}} \mathrm{X}_{\mathrm{i}+} \mathrm{X}_{+\mathrm{i}}}
$$

Donde $X_{i i}$ son los valores de la diagonal principal de la matriz, $\mathrm{X}_{i_{+}}$es el total marginal de la fila $\mathrm{i}, X_{+i}$ el total marginal de la columna $i$ y $N$ es el total de puntos de control como referencia. El valor mínimo estandarizado para la aceptación del producto final cartografiable obtenido por clasificación digital es del $85 \%$ de confiabilidad o exactitud global. Loya et al. (2013) sostienen que el coeficiente kappa es el parámetro estadístico que otorga mayor confiabilidad al producto final clasificado cuando este alcanza un valor mayor a 0,8. La escogencia del número mínimo de puntos de verificación se hizo mediante la expresión:

$$
\mathrm{n}=\frac{\mathrm{z}^{2} \mathrm{pq}}{\mathrm{E}^{2}}
$$

Donde $\mathrm{n}$ es el número mínimo de puntos de muestreo, $\mathrm{z}$ el factor de confiabilidad de acuerdo con el nivel de confianza en probabilidad, $p$ el mínimo de aciertos, $q$ el número mayor de desaciertos $q=1-p$ y $E$ el error estadístico. Se estimó el número mínimo de puntos a supervisar considerando una probabilidad del $95 \%$, un $z$ de 1,96 y error correspondiente al $5 \%$, con un mínimo de aciertos del $85 \%$ y en consecuencia un $15 \%$ desaciertos.

\section{Grupo hidrológico de suelos (HGS)}

La asignación de grupos hidrológicos de suelos se realizó mediante la clasificación textural de los distintos tipos de suelos reportados por IGAC (Instituto Geográfico Agustín Codazzi) como fuente oficial de información básica, teniendo en cuenta lo sugerido por NRCS (2009) y Mongil y Navarro (2012) en la asignación de grupos hidrológicos, considerando como referencia las composiciones más finas de granulometría del suelo por ser la condición más limitante a la infiltración de acuerdo con lo relacionado en la tabla 2. 
Tabla 2: Grupos hidrológicos de suelos (HGS). (Adaptado de NRCS, 2009)

\begin{tabular}{|l|l|l|l|}
\hline Textura & HGS & Textura & HGS \\
\hline Arenosa (Ar) & A & Franco-Arenosa (F-Ar) & $A^{*}-B$ \\
\hline Arenosa-franca (Ar-F) & $A^{*}-\mathrm{B}$ & Franco-arcillosa (F-a) & C \\
\hline Arcillosa (a) & $\mathrm{C}^{*}-\mathrm{D}$ & Franco-arcillo-arenosa (F-a-Ar) & $\mathrm{B}^{*}-\mathrm{C}$ \\
\hline Arcillo-arenosa (a-Ar) & $\mathrm{C}^{*}$ & Franco-arcillo-limosa (F-a-L) & $\mathrm{C}$ \\
\hline Arcillo-limosa (a-L) & $\mathrm{C}^{*}$ & Franco-limosa (F-L) & $\mathrm{A}^{*}-\mathrm{B}^{*}-\mathrm{C}$ \\
\hline Franca (F) & $\mathrm{A}^{*}-\mathrm{B}^{*}-\mathrm{C}$ & Limosa (L) & $\mathrm{B}^{*}$ \\
\hline
\end{tabular}

\section{Asignación de número de curva $(\mathrm{CN})$}

Se estimó el CN realizando el geoprocesamiento de intercepción entre la capa de los grupos hidrológicos del suelo HGS y la capa de coberturas para asignar el correspondiente CN de acuerdo con la condición de humedad antecedente de la zona.

\section{Precipitación de diseño y cálculo de escurrimiento potencial}

Se realizó el cálculo de escorrentía potencial con datos de precipitación máximas en 24 horas, estimadas con la información de las estaciones climatológicas de la zona de estudio, con períodos de retorno 2, 5, 10, 25, 50 y 100 años; utilizando el método predictivo de distribución de probabilidad de Gumbel descrito en Niemann y Diburg (2013). Para establecer la precipitación en el área, se usó la técnica de Polígonos de Thiessen mediante el Software ArcGIS.

\section{RESULTADOS Y DISCUSIÓN}

El análisis de las variables climáticas de temperatura, evapotranspiración potencial y densidad de vapor se realizó para las estaciones Media Luna, Normal Manatí, y Repelón ubicadas en la zona Norte y las estaciones El guamo y Los Álamos ubicadas en la zona Sur, al ser las únicas dotadas para medir las variables climáticas requeridas en la investigación. En la tabla 3 se presentan los valores promedios de temperatura, evapotranspiración y densidad de vapor en el área de estudio. En términos generales, en las 5 estaciones mencionadas se encontró que las variables presenten una tendencia de aumento. Mediante la línea de regresión de mejor ajuste a la serie histórica de datos, se calcularon los valores de las variables de estudio para los años 1985 y 2016; encontrando que las estaciones Media Luna, Normal Manatí y Repelón, la temperatura ha presentado aumentos de $0.6^{\circ} \mathrm{C}, 0.4^{\circ} \mathrm{C}$ y $0.8^{\circ} \mathrm{C}$ respectivamente, lo cual implica que para el periodo de análisis se presenta una variación promedio de $0.6^{\circ} \mathrm{C}$. Para las estaciones Los Alamos y El Guamo se obtuvieron aumentos de $1^{\circ} \mathrm{C}$ y $0,5^{\circ} \mathrm{C}$ respectivamente, siendo en la zona sur el aumento más notorio de temperatura. Estas tendencias no desconocen los patrones de variabilidad climática en el área de estudio; los cuales son un indicativo de cambios en el clima local.

Tabla 3. Valores promedio de T, ETP y Dv en la zona de estudio

\begin{tabular}{|c|c|c|c|}
\hline \multirow{2}{*}{ Estación } & \multicolumn{3}{|c|}{ Promedio } \\
\cline { 2 - 4 } & $\mathrm{T}\left({ }^{\circ} \mathrm{C}\right)$ & $\mathrm{Dv}(\mathrm{g} / \mathrm{m} 3)$ & $\mathrm{ETP}(\mathrm{mm})$ \\
\hline Media Luna & 28.1 & 22.5 & 1412.2 \\
\hline Normal Manati & 27.8 & 22.2 & 1397.8 \\
\hline Repelón & 28.5 & 21.7 & 1431.6 \\
\hline Los Álamos & 27.9 & 22.1 & 1387.2 \\
\hline El Guamo & 27.7 & 19.9 & 1386.1 \\
\hline
\end{tabular}

En la zona de estudio se encontró una estrecha relación entre la ocurrencia de los fenómenos de El Niño y La Niña con los valores de temperatura mensual en uno o más meses por fuera de lo normal (valores de $T$ entre el promedio y +/- la desviación estándar). La ocurrencia de estos eventos genera enfriamientos o calentamientos en la atmósfera regional, de manera que tiene influencia en el comportamiento de variables climáticas en la denominada zona intertropical del Ecuador (Poveda y Mesa 1999; Zveryaev y Allan 2005). Aun cuando la zona de estudio se ve influenciada por los fenómenos de variabilidad climática, se ha mantenido la tendencia de aumento de la temperatura del aire en los 32 años de registro existentes.

El aumento de temperatura se ve reflejado en el aumento paulatino de la evapotranspiración potencial de la interface suelo - vegetación, donde se encontraron aumentos de $34.3 \mathrm{~mm}, 15.7 \mathrm{~mm}$ y $35.3 \mathrm{~mm}$ para cada una de las estaciones en cuestión (Media Luna, Normal Manatí y Repelón respectivamente), y aumentos de $49.1 \mathrm{~mm}$ y $20.6 \mathrm{~mm}$ para las estaciones La Álamos y El Guamo, lo cual podría estar compensando el aumento 
de precipitaciones que se presenta en la zona. Al existir más agua disponible en la interfase suelo-vegetación como consecuencia de una mayor cantidad de lluvia precipitada, es también mayor la cantidad de agua evaporada posteriormente desde la superficie hacia la atmosfera, por efecto de la radiación solar incidente (Pérez y Gónima 2014).

Como una aproximación a las variaciones que pueda presentar la humedad atmosférica mediante el cálculo de la densidad de vapor, se encontró en el período de análisis un aumento de $0.6 \%, 2.2 \%$ y $15.7 \%$ para las estaciones Media Luna, Normal Manati, y Repelon; mientras que para las estaciones Los Alamos y El Guamo se obtuvieron aumentos del $1.7 \%$ y $5.8 \%$; lo cual indica que existe mayor concentración de vapor de agua en la atmósfera que podría estar contribuyendo en los aumentos de la temperatura local (Dessler, Zhang, y Yang 2008; Pérez y Gónima 2014; Zveryaev y Allan 2005). En la tabla 4 se resume las diferencias calculadas y los cambios porcentuales de las variables estudiadas, de igual manera en la figura 2 se presentan de forma cualitativa las variaciones de dichas variables a través de los años; con su respectiva ecuación de tendencia de mejor ajuste.

Tabla 4: Variación cuantitativa de las variables climáticas en el periodo de estudio

\begin{tabular}{|c|c|c|c|c|c|c|}
\hline \multirow{2}{*}{ Estación } & \multicolumn{2}{|c|}{$\mathrm{T}\left({ }^{\circ} \mathrm{C}\right)$} & \multicolumn{2}{c|}{$\mathrm{Dv}\left(\mathrm{g} / \mathrm{m}^{3}\right)$} & \multicolumn{2}{c|}{$\mathrm{ETP}(\mathrm{mm})$} \\
\cline { 2 - 7 } & $\Delta \mathrm{T}\left({ }^{\circ} \mathrm{C}\right)$ & $\Delta \mathrm{T}(\%)$ & $\Delta \mathrm{Dv}\left(\mathrm{g} / \mathrm{m}^{3}\right)$ & $\Delta \mathrm{Dv}(\%)$ & $\Delta \mathrm{ETP}(\mathrm{mm})$ & $\Delta \mathrm{ETP}(\%)$ \\
\hline Media Luna & 0.6 & 2.3 & 0.1 & 0.6 & 34.3 & 2.4 \\
\hline Normal Manati & 0.4 & 1.3 & 0.5 & 2.2 & 15.7 & 1.1 \\
\hline Repelón & 0.8 & 2.7 & 3.5 & 15.9 & 35.3 & 2.5 \\
\hline Los Álamos & 1.0 & 3.4 & 0.4 & 1.7 & 49 & 3.5 \\
\hline El Guamo & 0.5 & 1.9 & 1.2 & 5.8 & 20.6 & 1.5 \\
\hline
\end{tabular}

\section{Determinación de la cobertura vegetal}

La determinación de la cobertura vegetal se realizó para la discriminación de bosque, cultivo permanente, cuerpos de agua, zona pantanosa, pasto (para el cual se discriminó entre bueno y pobre), nubes, sombras, suelo desnudo y zona urbana. El factor de índice óptimo de mayor valor correspondió a la combinación RGB 567 con magnitud de 6567.97 sobre la cual se realizó la agrupación de las clases. La validación de la clasificación mediante la matriz de confusión, se realizó analizando 208 puntos de interés, superior al número mínimo de puntos de verificación (196 puntos), empleando imágenes de mayor resolución de geovisores como Earth Explorer y Google Earth, entre otros y datos verificados directamente en campo para aquellas áreas con mayor incertidumbre. Se obtuvo una confiabilidad global del $90.38 \%$ y un coeficiente Kappa de 0.8552, aprobándose con esto los resultados obtenidos en la clasificación.

De acuerdo con los resultados de la clasificación supervisada, en once de las microcuencas estudiadas, más del $40 \%$ de su territorio está cubierto por pastos, convirtiéndose en la cobertura predominante en el área de estudio (604863.4 ha), siendo la condición de pasto pobre la más extensa (547104.6 ha). El 58\% de la extensión del municipio de Sitio Nuevo (52176.1 ha) se encuentra cubierto por zonas pantanosas o aguas continentales, convirtiéndolo en un municipio muy susceptible a las inundaciones periódicas debido su baja capacidad de regulación hídrica. El 61\% del territorio de la microcuenca del rio Sevilla (66589 ha) se encuentra cubierto por bosque denso, debido a que su parte alta está ubicada en la Sierra Nevada de Santa Marta en un sector poco intervenido por la acción antrópica. Una gran extensión del territorio de la microcuenca del rio Sevilla y de la microcuenca de la Quebrada La Aguja están fuertemente antropizadas, considerando que el $15.5 \%$ (16882.2 ha) y el $27 \%$ (2476 ha) de su territorio respectivamente; están cubiertos por cultivos permanentes, principalmente Banano, lo cual hace que las microcuencas sean muy vulnerables a la contaminación por agroquímicos utilizados en este tipo de plantaciones.

La cobertura vegetal predominante de pastos, no favorece los procesos de regulación hídrica y térmica como procesos fundamentales desarrollados por la vegetación (arbórea mayormente) de acuerdo con lo enunciado en FAO (2003). Posiblemente, la escasa cubierta vegetal del terreno puede estar influyendo en los procesos de calentamiento rápido del suelo y con ello el calentamiento de la superficie por transferencia de calor, al quedar expuesto a la radiación solar, permitiendo que el agua contenida en los perfiles superficiales se evapore con facilidad, lo cual puede presentar una condición seca de los mismos en periodos cortos de tiempo. Esta condición, en términos hidrológicos, no favorece el proceso de infiltración de agua en el suelo, potencializando el escurrimiento, dado que la cobertura es una de las variables de mayor influencia en el escurrimiento superficial, como respuesta hidrológica del territorio. En la tabla 5 se resumen las distintas coberturas discriminadas y su extensión en el área de estudio. 



Fig. 2: Tendencia multianual de T, ETP y Dv en la zona de estudio. 
Tabla 5: Cobertura del terreno en el área de estudio

\begin{tabular}{|l|c|c|}
\hline Cobertura & Área (ha) & Fracción (\%) \\
\hline Agua & 52681.38 & 4.96 \\
\hline Bosque & 310839.60 & 29.28 \\
\hline Cultivo permanente & 22881.83 & 2.16 \\
\hline Nube & 1917.52 & 0.18 \\
\hline Pasto & 604863.40 & 56.99 \\
\hline Sombra & 1193.53 & 0.11 \\
\hline Suelo desnudo & 30477.66 & 2.87 \\
\hline Tejido urbano & 3169.49 & 0.30 \\
\hline Zona pantanosa & 33417.19 & 3.15 \\
\hline Total & 1061441.60 & 100 \\
\hline
\end{tabular}

\section{Grupo hidrológico de suelos (HSG)}

La estimación de grupos hidrológicos de suelos tuvo como insumo base la clasificación textural realizada por IGAC (2009) con escala 1:100.000, teniendo en cuenta el horizonte con mayor porcentaje de limos y arcillas como condición limitante a la infiltración, considerando que la composición granulométrica del suelo no varía en periodos cortos de tiempos. La tabla 6 muestra la respectiva asignación de grupos hidrológicos para la extensión total del área de estudio, teniendo en cuenta lo sugerido por Mongil y Navarro (2012) y NRCS (2009).

Tabla 6: Asignación de HGS

\begin{tabular}{|c|c|c|}
\hline HSG & Área (ha) & Fracción (\%) \\
\hline A & 25860.50 & 2.4 \\
\hline B & 21016.35 & 19.8 \\
\hline C & 727084.98 & 68.5 \\
\hline D & 23104.83 & 2.2 \\
\hline Agua & 75254.57 & 7.1 \\
\hline Total & 1061521.22 & 100 \\
\hline
\end{tabular}

En esta clasificación de grupos hidrológicos se asigna la categoría D a la superficie de zona urbana (ZU) por presentar alto grado de impermeabilización y por tanto afectación considerable a la infiltración (NRCS, 2009). En diez de las microcuencas de estudio más del $70 \%$ de su territorio se encuentra dentro de la clasificación de grupo hidrológico $\mathrm{C} \circ \mathrm{D}$, lo que deja como evidencia que por las condiciones naturales de los suelos se dificultan la infiltración de la lluvia, potenciando así el escurrimiento. El $81.5 \%$ y el $77.4 \%$ de los suelos en las microcuencas de la quebrada La Aguja y Rio Sevilla respectivamente, se clasifican en un grupo hidrológico A ○ B, convirtiéndolas en las microcuencas que mejor favorecen los procesos de infiltración por las condiciones naturales de sus suelos. El $57.4 \%$ de los suelos del municipio de Sitio Nuevo se clasifican dentro de un grupo hidrológico C o D, y el $46.6 \%$ restante se encuentra cubierto por agua lo que lo convierte en el territorio que no favorece en absoluto la infiltración.

\section{Asignación de número de curva $(\mathrm{CN})$}

Una vez asignados los grupos hidrológicos de suelos y realizada la verificación del producto final de la clasificación de cobertura vegetal, se realiza la intercepción de las capas de HSG y de cobertura vegetal, realizando el cálculo del CN, teniendo en cuenta que el área de estudio presenta un AMC III, por lo cual se ajustó a dicha condición de humedad antecedente. A los tipos de coberturas de agua, suelo desnudo y tejido urbano se les asigno un $\mathrm{CN}$ de 100 suponiendo una afectación total a la infiltración. Los resultados del cálculo del CN, evidencian que once de las microcuencas de estudio presentan números de curva mayores de 80 en más del $85 \%$ de su territorio, lo que indica la alta afectación de la capacidad de infiltración, lo que se traduce en altos escurrimientos sobre el valor total de la precipitación que llega a la superficie. El $23.5 \%$ (2147.8 ha) y el $44.3 \%$ (58749.2 ha) del territorio de las microcuencas de la Quebrada La Aguja y Rio Sevilla respectivamente, presentan números de curva menores a 80 lo que indica que estas microcuencas presentan una mejor capacidad de infiltración y son menos propensas a crecientes súbitas por eventos extremos de precipitación. En la figura 3 se presenta la distribución espacial del CN calculado para la zona de estudio. 


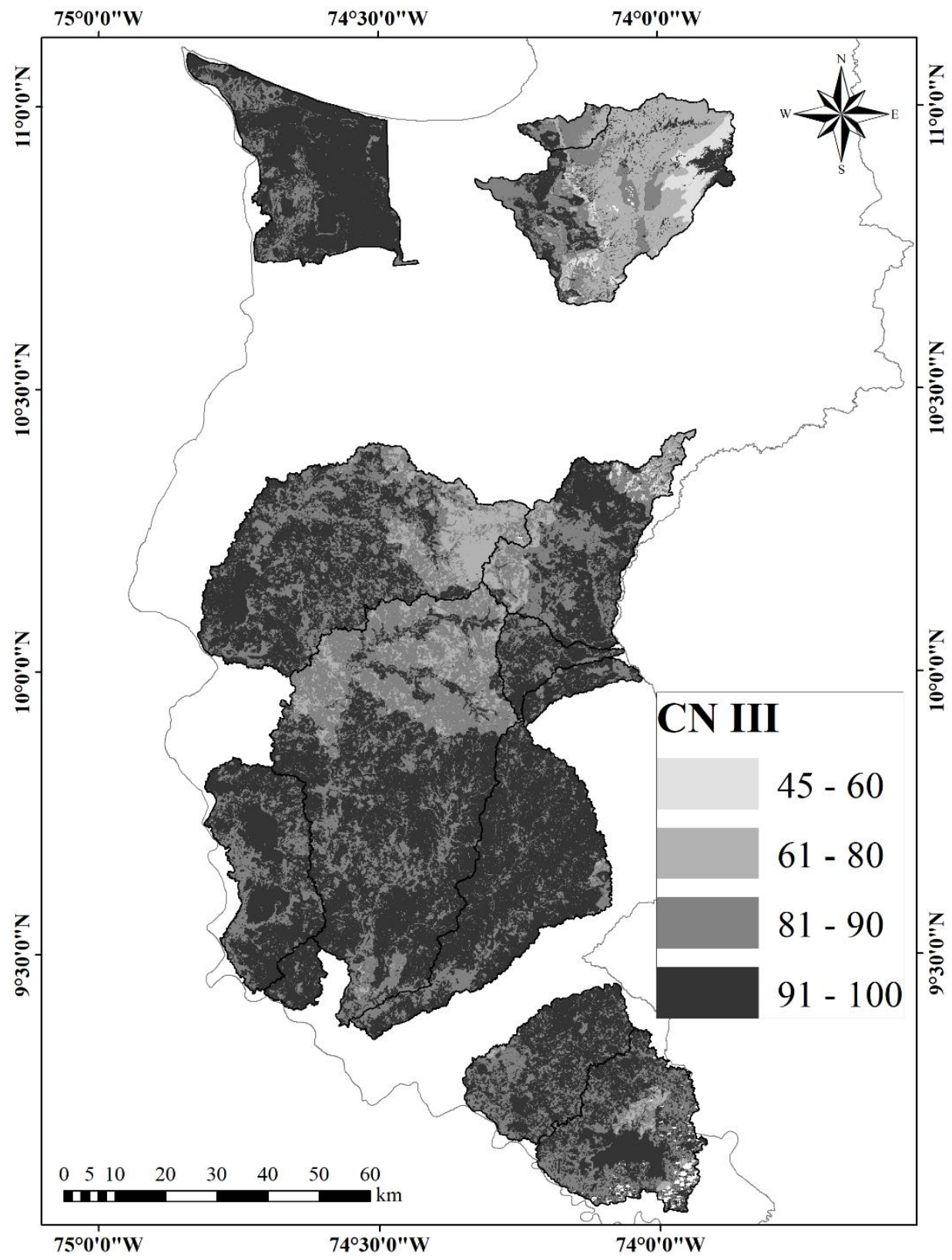

Fig. 3: Mapa de número de curva $(\mathrm{CN})$ en el área de estudio.

\section{Precipitación de diseño y escurrimiento potencial}

Mediante la distribución probabilística de Gumbel para valores extremos se estimó las precipitaciones máximas en 24 horas con periodos de retorno de 2, 5, 10, 25, 50 y 100 años para cada estación de interés. Se encontró que cada dos años pueden ocurrir escurrimientos mayores de $75 \mathrm{~mm}$ entre el $64 \%$ y el $87.9 \%$ del territorio en las microcuencas de las ciénagas La Rinconoda y EI Sapo respectivamente, y valores mayores de $100 \mathrm{~mm}$ cada 5 años en un porcentaje del $47.4 \%$ y el $87.9 \%$ del territorio de estas microcuencas, lo cual indica que presentan una baja capacidad de infiltración de la lluvia por las condiciones de la cobertura vegetal y el tipo de suelo. Lo anterior permite inferir que estas microcuencas presentan una alta vulnerabilidad ante crecientes súbitas y procesos erosivos como resultado de los eventos extremos de precipitación. Las microcuencas Quebrada La Aguja y Rio Sevilla presentan los menores valores de escurrimientos potenciales teniendo en cuenta que cada 10 años podrían ocurrir escurrimientos mayores a $100 \mathrm{~mm}$ solo en $5.3 \%$ a $9.5 \%$ de su territorio. Las microcuencas de la ciénaga Zapayan, arroyo Cacagüero, Melchorero y Torres poseen valores altos de numero de curva, pero cada 10 años solo se esperarían escurrimientos mayores a $100 \mathrm{~mm}$ en un área no superior al $4.5 \%$ de su territorio, esto se debe principalmente a los valores de precipitación de esta zona.

Las estimaciones de los eventos máximos en 24 horas realizadas con el método de Gumbel no arrojaron lluvias de diseño extremas para un periodo de retorno de diez años, pero al aumentar el periodo de retorno, 
aumenta la lluvia de diseño y por ende los escurrimientos potenciales. Los altos valores de escurrimientos potenciales para eventos extremos de precipitación son un reflejo de las limitaciones naturales del territorio establecidas por el tipo de textura del suelo y la cobertura vegetal, siendo la cobertura de pastos la predominante en gran parte del área de estudio.

Teniendo en cuenta que gran parte de los volúmenes de lluvia se pierden por escurrimiento, es inherente se afecte la disponibilidad de agua en las capas superficiales del suelo; al igual la recarga de acuíferos. La cobertura de pastos no favorece los procesos de abstracción y por tanto de regulación hídrica; coadyuvando en el calentamiento de la superficie. Los altos escurrimientos representan un factor de riesgo inminente para el territorio, pudiéndose reflejar en el aumento de los procesos erosivos y con ello degradación del suelo, crecientes súbitas, inundaciones, remociones en masa, entre otros. En tal sentido, es indispensable que los cuerpos de agua superficial (corrientes y humedales) tengan la capacidad hidráulica para la recepción y transporte de los volúmenes generados por escorrentía. En la figura 4 se presenta la distribución espacial de los escurrimientos potenciales en la zona de estudio para lluvias con diferentes periodos de retorno.

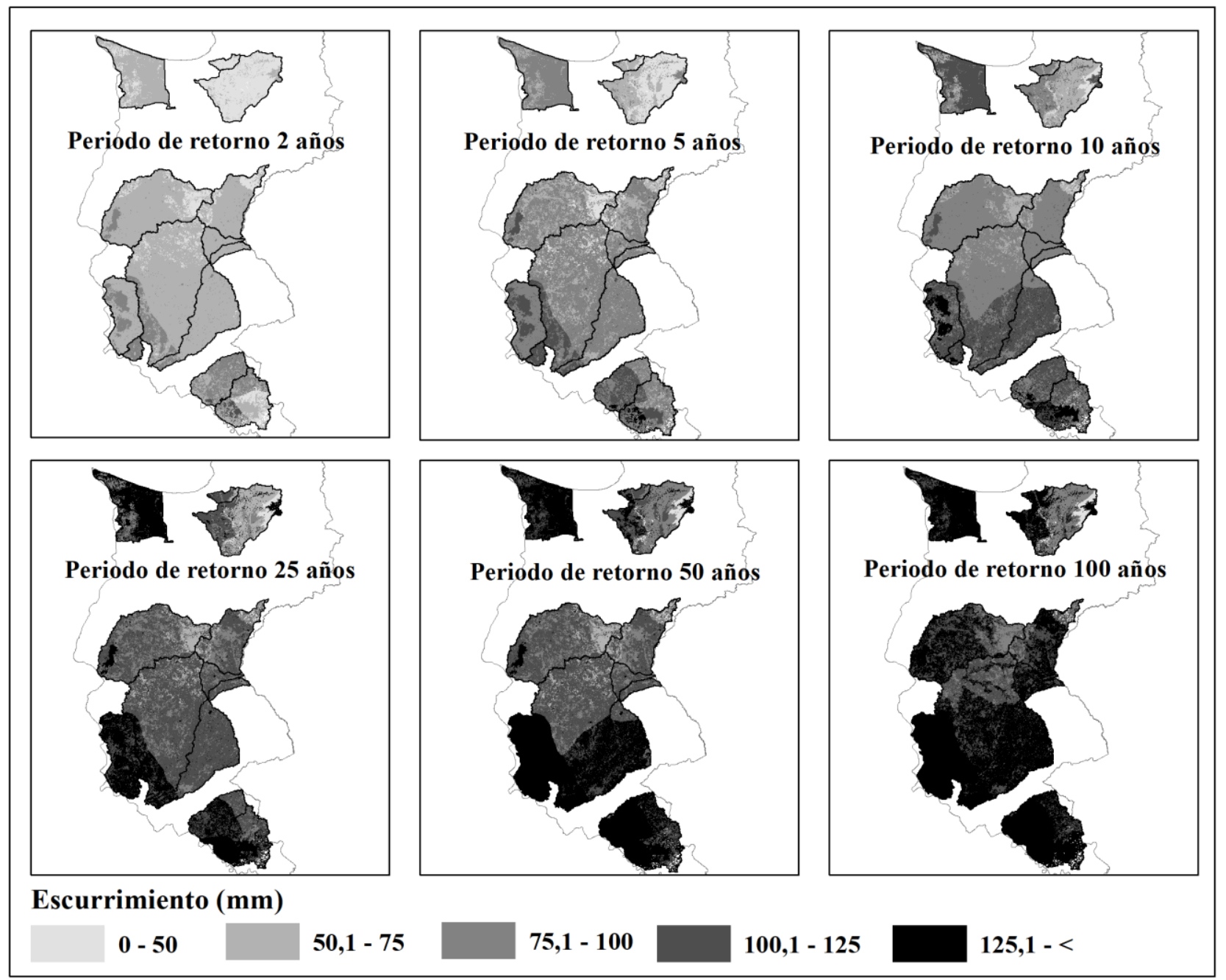

Fig. 4: Distribución espacial de los escurrimientos potenciales en la zona de estudio para lluvias con diferentes periodos de retorno

\section{CONCLUSIONES}

De acuerdo a los resultados obtenidos en la investigación se pueden extraer las siguientes conclusiones:1) La temperatura del aire presenta aumentos entre 1.3 a 3.4\% para la zona de estudio, con diferencias entre las estaciones climáticas; 2) la evapotranspiración potencial como aproximación al complejo suelo vegetación muestra tendencias de aumento entre 1.1 a $3.5 \%$; 3) la densidad de vapor de agua muestra tendencia de aumento en un $0.6 \%$ y $15.9 \%$ con diferencias entre las estaciones, corroborando el rol de este gas como indicador de calentamiento a escala local para zonas con temperatura y humedad relativa alta; 4) el clima local está influenciado por los fenómenos oscilatorios El Niño y La Niña producidos en el pacífico Sur; pero a pesar de ello se ha mantenido la tendencia de aumento en la temperatura del aire en los treinta y dos años de registro analizados; 5) la cobertura vegetal del territorio no favorece la retención de humedad en el 
suelo ni el equilibrio hídrico a través de la abstracción inicial al perderse gran cantidad de la lluvia por escurrimiento, coadyuvando posiblemente en el calentamiento local; 6) el grupo hidrológico del suelo predominante fue $\mathrm{C}$ con una extensión del $68.5 \%$ en la zona de estudio, la cual limita el proceso de infiltración y favorece la tendencia de aumento del escurrimiento y 7) las tendencias de posible cambio en el clima local, están relacionadas con el estado físico del territorio en términos de cobertura vegetal como principal regulador hídrico y térmico. Este escenario afecta la disponibilidad de agua para los distintos usos y procesos fisiológicos vegetales, aun cuando la oferta por precipitación sea de tendencia al aumento.

\section{REFERENCIAS}

Alarcón, J., Zafra, C., y Echeverri, L., Cambio climático y recursos hídricos en Colombia, https://doi.org/http://doi.org/10.31910/rudca.v22.n2.2019.1368, Actualidad y Divulgación Científica, 22(2), 1-10, (2019).

Bryson, B., Zbyszek, K., Shaohong, W., y Jean, P., Climático y el Agua. Ginebra, (2008).

Camuffo, D., Microclimate for Cultural Heritage. Agricultural and Forest Meteorology, 111, 309, (2002).

Correia, F.W., Alvala, R.C., y Manzi, A.O. Modeling the impacts of land cover change in Amazonia: a regional climate model (RCM) simulation study, https://doi.org/10.1007/s00704-007-0335-z, Theorical and Applied Climatology, 93, 225244, (2008).

Costa, C., La adaptación al cambio climático en Colombia, Revista de Ingeniería de La Universidad de Los Andes, 26, 74-80, (2007).

Dessler, A.E., Zhang, Z., y Yang, P. Water-vapor climate feedback inferred from climate fluctuations, 2003-2008, https://doi.org/10.1029/2008GL035333, Geophysical Research Letters, 35(20), 10-13, (2008).

FAO, Programa de lucha contra el hambre; Enfoque de doble componente para la reducción del hambre: prioridades para la actuación a nivel nacional e internacional, Roma, (2003).

FAO, Evapotranspiración del cultivo: Guías para la determinación de los requerimientos de agua de los cultivos, (FAO, Ed.), Roma, (2006).

Gónima, L., Evidencias del calentamiento global en el Caribe Colombiano. Meteorologia Colombiana 13, 1-10, (2009).

Gónima, L., y Pérez, M., Analisis de la variabilidad climática de la temperatura del aire y de la ETP para una zona del Caribe Colombiano, Meteorología Colombiana, 1, 183-195, (2012).

Intergovernamental Panel on Climate Change - IPCC. IPCC Fourth Assessment Report: Climate Change 2007, 3-4, (2007).

Kousari, M.R., Ekhtesasi, M.R. y otros 3 autores, An investigation of the Iranian climatic changes by considering the precipitation, temperature, and relative humidity parameters, https://doi.org/10.1007/s00704-010-0304-9, Theorical and Applied Climatology, 103, 321-335, (2011).

Leckner, B., The spectral distribution of solar radiation at the earth's surface-elements of a model, https://doi.org/10.1016/0038-092X(78)90187-1, Solar Energy, 20(2), 143-150, (1978).

Loya, J.O., Aguilar, S.A., Bravo, L.C., y Sánchez, E., Evaluación espacio-temporal del impacto del crecimiento urbano sobre la cobertura vegetal en la región fronteriza Nogales, México y Arizona, Estados Unidos, durante el periodo 19952010, Revista Latinoamericana de Recursos Naturales, 9(1), 124-140, (2013).

Mongil, J., y Navarro, J., Infiltración y grupos hidrológicos de suelos en las laderas de los páramos (Valladolid), Cuadernos de Investigacion Geografica, 38(1), 131-153, (2012).

Napoli, M., Massetti, L., y Orlandini, S. Hydrological response to land use and climate changes in a rural hilly basin in Italy, https://doi.org/10.1016/j.catena.2017.05.002, Catena, 157, 1-11, (2017).

New, M., Hulme, M., y Jones, P., Representing Twentieth-Century Space - Time Climate Variability . Part I : Development of a $1961-90$ Mean Monthly Terrestrial Climatology, https://doi.org/http://dx.doi.org/10.1175/15200442(1999)012<0829:RTCSTC>2.0.CO;2, American Meteorological Society, 12, 829-856, (1999).

New, M., Hulme, M., y Jones, P., Representing Twentieth-Century Space - Time Climate Variability . Part II : Development of 1901 - 96 Monthly Grids of Terrestrial Surface Climate, https://doi.org/http://dx.doi.org/10.1175/15200442(2000)013<2217:RTCSTC>2.0.CO;2, American Meteorological Society, 2217-2238, (2000).

Niemann, H.J., y Diburg, S., Statistics of extreme climatic actions based on the gumbel probability distributions with an upper limit, https://doi.org/10.1016/j.compstruc.2013.03.016, Computers and Structures, 126(1), 193-198, (2013).

NRCS, Hydrology National Engineering Handbook, Chapter 7: Hydrologic Soil Groups, (2009).

Pérez, M., y Gónima, L., El contenido del Vapor de agua de la atmósfera como indicador de calentamiento global en una zona del Caribe de Colombia, Cuadernos de Investigación Geográfica, 40(2), 477-495, (2014).

Poveda, G., y Mesa, O., La Corriente de Chorro Superficial del Oeste (“ del CHOCÓ ") y otras dos corrrientes de chorro en Colombia sobre: climatología y variabilidad durante las fases del ENSO, Revista Académica Colombiana de Ciencia, 23(89), 517-528, (1999). 
Roberto, A., y Oregon, W., Adaptation of the Thornthwaite scheme for estimating daily reference evapotranspiration, https://doi.org/10.1016/j.agwat.2003.11.003, Agricultural Water Management, 66, 251-257, (2004).

Shiferaw, H., Gebremedhin, A., Gebretsadkan, T., y Zenebe, A., Modelling hydrological response under climate change scenarios using SWAT model : the case of Ilala watershed, Northern Ethiopia, https://doi.org/10.1007/s40808-018-04398, Modeling Earth Systems and Environment, 4, 437-449, (2018).

Zabaleta, A., Calentamiento global a escala local y disponibilidad hídrica en los municipios de Planeta Rica, Sahagún y Chinú, Córdoba, Colombia, Trabajo de grado Ingeniero Ambiental, Facultad de Ingenierías, Universidad de Córdoba. Montería, Colombia. 116 págs, (2017).

Zabaleta, A., Mercado, T., Marrugo, J. L., y Feria, J., Curve Number ( CN ) as Pressure Indicator of the Hydrological Condition under Global Warming Scenarios at a Local Scale in La Mojana Region , Colombia, https://doi.org/10.17485/ijst/2018/v11i29/129276, Indian Journal of Science and Technology, 11(29), 1-12, (2018).

Zhang, C., Wang, X., Li, J., y Hua, T. Identifying the effect of climate change on desertification in northern China via trend analysis of potential evapotranspiration and precipitation. https://doi.org/10.1016/j.ecolind.2020.106141, Ecological Indicators, 112, (2020).

Zveryaev, I.I., y Allan, R.P., Water vapor variability in the tropics and its links to dynamics and precipitation, https://doi.org/10.1029/2005JD006033, Journal of Geophysical Research Atmospheres, 110(21), 1-17, (2005). 
